\title{
Equilibrium probability distribution for number of bound receptor-ligand complexes
}

Tuhin Chakrabortty, and Manoj M. Varma

Citation: American Journal of Physics 89, 41 (2021); doi: 10.1119/10.0001898

View online: $\mathrm{https}: / /$ doi.org/10.1119/10.0001898

View Table of Contents: https://aapt.scitation.org/toc/ajp/89/1

Published by the American Association of Physics Teachers

\section{ARTICLES YOU MAY BE INTERESTED IN}

Six textbook mistakes in computational physics

American Journal of Physics 89, 51 (2021); https://doi.org/10.1119/10.0001945

Rabi oscillations, Floquet states, Fermi's golden rule, and all that: Insights from an exactly solvable two-level model

American Journal of Physics 89, 26 (2021); https://doi.org/10.1119/10.0001897

Neumann, but hold the von

American Journal of Physics 89, 9 (2021); https://doi.org/10.1119/10.0002857

A vertical race up and back down with and without drag

American Journal of Physics 89, 67 (2021); https://doi.org/10.1119/10.0001893

High-speed escape from a circular orbit

American Journal of Physics 89, 72 (2021); https://doi.org/10.1119/10.0001956

An optical n-body gravitational lens analogy

American Journal of Physics 89, 11 (2021); https://doi.org/10.1119/10.0002117

AAPT,

Advance your teaching and career as a member of AAPT 


\title{
Equilibrium probability distribution for number of bound receptor-ligand complexes
}

\author{
Tuhin Chakrabortty \\ Center for Nano Science and Engineering, Indian Institute of Science, Bangalore 560012, India \\ Manoj M. Varma \\ Center for Nano Science and Engineering, Indian Institute of Science, Bangalore, India and \\ Robert Bosch Centre for Cyber-Physical Systems, Indian Institute of Science, Bangalore 560012, India
}

(Received 23 January 2020; accepted 19 August 2020)

\begin{abstract}
The phenomenon of molecular binding, where two molecules, referred to as a receptor and a ligand, bind together to form a ligand-receptor complex, is ubiquitous in biology and essential for the accurate functioning of all life-sustaining processes. The probability of a single receptor forming a complex with any one of $L$ surrounding ligand molecules at thermal equilibrium can be derived from a partition function obtained from the Gibbs-Boltzmann distribution. We extend this approach to a system consisting of $R$ receptors and $L$ ligands to derive the probability density function $p(r ; R, L)$ to find $r$ bound receptor-ligand complexes at thermal equilibrium. This extension allows us to illustrate two aspects of this problem which are not apparent in the single receptor problem, namely, (a) a symmetry to be expected in the equilibrium distribution of the number of bound complexes under exchange of $R$ and $L$ and (b) the number of bound complexes obtained from chemical kinetic equations has an exact correspondence to the maximum probable value of $r$ from the expression for $p(r ; R, L)$. We derive the number fluctuations of $r$ and present a practically relevant molecular sensing application which benefits from the knowledge of $p(r ; R, L)$. (C) 2021 American Association of Physics Teachers.
\end{abstract}

https://doi.org/10.1119/10.0001898

\section{INTRODUCTION}

The phenomenon of molecular binding, where two molecules, generally referred to as a receptor and a ligand, bind together to form a ligand-receptor complex, occurs ubiquitously in biological systems. This phenomenon is essential for the accurate functioning of many life-sustaining processes ranging from DNA transcription to immune response. ${ }^{1,2}$ The ligand-receptor complex formation is a reversible process, which means that while a ligand molecule can associate with a receptor molecule to form a ligandreceptor complex, the converse process, namely, the dissociation or breaking down of a ligand-receptor complex into one each of the ligand and receptor molecules, can also happen simultaneously. In general, the rates of these two competing processes would be different leading to one process dominating over the other. As we will see in Sec. II of this article, thermodynamic equilibrium is reached when the rates of these two processes become equal. The equilibrium state is also a steady state where the number of the bound ligandreceptor complexes remains constant over time due to the two equal and opposite processes cancelling each other. Living organisms are non-equilibrium systems, which exchange energy and matter with the surroundings, as illustrated beautifully by Rob Philips. ${ }^{3}$ The separation of time-scales allows a large number of biological processes involving molecular binding to be viewed as processes in equilibrium. Due to this ubiquitous and critical role of molecular binding in biological functions, we would like to be able to answer questions such as how many ligandreceptor complexes do we expect to find at equilibrium after we start with $R$ receptors and $L$ ligands. As we will see in the next paragraph, there are a number of different ways to answer this question and related questions such as the magnitude of fluctuations in the number of ligand-receptor complexes at equilibrium.

One of the ways to answer the question on the number of ligand-receptor complexes to be expected at equilibrium, starting from $R$ receptors and $L$ ligands, is by writing down the rate equations by invoking the law of mass action. ${ }^{4}$ This approach is worked out in Sec. II of this article. This approach provides not only the number of ligand-receptor complexes at equilibrium but also how the number of complexes changes as a function of time from the initial value of zero. A question then arises, namely, if we perform $N$ independent experiments each with $R$ receptors and $L$ ligands and measure the number of ligand-receptor complexes at equilibrium for each of these experiments, will we always get the same number as predicted by the reaction rate approach? As we might expect, the answer is no. There will be variation in the number of ligand-receptor complexes seen in the different experiments, which can be quantitatively characterized by the variance or standard deviation of the measured values. The variation in the measured number of complexes arises not just because of experimental uncertainties but also due to a more fundamental reason. The binding of a receptor and a ligand molecule to form the complex as well as its dissociation at a later point in time are stochastic events which follow certain probability distributions. As a result, even if the experimental uncertainties were reduced to zero hypothetically, one would still find a distribution of values for the number of ligand-receptor complexes even if one performed identical experiments repeatedly. Therefore, an estimate for the magnitude of the variation is essential to obtain a fundamental understanding of noise in biological systems $^{5-8}$ or in a more applied context to understand and optimize the performance of biosensors. Biosensors are devices which rely on molecular binding to detect or quantify the 
presence of specific molecules in human samples such as blood. The amount of these specific molecules, referred to as biomarkers, indicate the presence of diseases such as cancer. ${ }^{9}$ One may like to know the fluctuations in the biosensor signal caused due to the stochastic nature of molecular binding in diagnostic applications, which are generally performed under equilibrium conditions. The magnitude of these fluctuations sets a fundamental limit on the minimum detectable number of biomarkers. As we will see in Sec. II, the reaction rate approach can only tell us the expected number of ligand-receptor complexes but not its fluctuations. In order to calculate these fluctuations, one needs to write what is referred to as a master equation which describes the temporal evolution of the different states of a system by accounting for the transitions into and out of the respective states. ${ }^{10}$ The solution of the master equation gives us the complete probability distribution of occupancy of the various states of a system, including at thermal equilibrium. From this equilibrium probability distribution, we can obtain the expected value, variance, and other higher moments. In fact, we can also obtain the rate equation (Eq. (1), described in Sec. I) from the master equation. ${ }^{11}$ Master equations are sometimes difficult to solve and, as an alternative, one can use the Gibbs-Boltzmann distribution if only the equilibrium probability distribution is sought after. We will follow this method to obtain the equilibrium probability distribution of the number of receptor-ligand complexes.

We know that equilibrium statistical mechanics allows us to calculate the distribution of particles into the various states of a given system. These states may be discrete or continuous and are characterized by a specific energy level of the state. By enumerating all the states of a given system appropriately scaled by their thermodynamic weights, one can obtain the partition function of the system which then yields the probability distribution of particles in these states. In the molecular binding problem, one could consider the unbound ligand and receptor molecules as one state and the bound complex as another state forming a two-state system and calculate the partition function. This approach has been exactly followed to derive the equilibrium occupation probability of a single receptor surrounded by $L$ ligand molecules. $^{3,6,12,13}$ The equilibrium occupation probability of the receptor is the probability that the receptor will be bound by one of the $L$ ligand molecules in equilibrium. The resultant formula is shown to be equivalent to the one which is obtained from the chemical reaction rate equations. ${ }^{14}$ This problem serves as a good illustration of how statistical mechanics links macroscopic parameters such as the equilibrium dissociation constant to the microscopic, molecular level details such as the energy-level difference between bound and unbound states.

Although the problem with one receptor and $L$ ligands serves to illustrate the application of equilibrium statistical mechanics to solve the binding problem, in many real situations we have more than one receptor. For instance, a cell may have multiple receptors on its surface and a biosensor certainly has millions of receptors which are used to bind a ligand target. Therefore, we would like to extend the solution of the one receptor problem into one which has $R$ receptors. The formal statement of the problem is to calculate the probability distribution of the number of receptor-ligand complexes $r$, under thermal equilibrium conditions, given $R$ receptors and $L$ ligands. If the binding of ligands to receptors can be considered as independent events, then the answer is simply $R$ times the probability of binding a single receptor by $L$ ligands which has been solved previously. The assumption of independence is a little tricky, because the occupation of one receptor by a ligand implies that for the next complex formation there is one less receptor and one expects that the probabilities of binding would change. However, are there situations where independence of events can be justified? To answer such questions systematically, one way forward is to write the partition function for the system and obtain the required probability distribution.

In this article, we derive the partition function by direct enumeration and from it the probability density function $p(r ; R, L)$, which is the probability to find $r$ bound receptorligand complexes at thermal equilibrium with the initial receptor and ligand concentrations of $R$ and $L$, respectively. The article is divided into the following sections: In Sec. II, we derive the number of bound complexes $r_{e q}$ at equilibrium using the conventional approach based on balancing of forward and backward reaction rates. Here, we show that the expression for the number of bound receptors from the binding kinetic equation $\left(b_{e q}\right)$ is symmetric with respect to the exchange of $R$ and $L$ and discuss why this symmetry is to be expected in this problem. In Sec. III, we present the partition function for a system consisting of $R$ receptors and $L$ ligands which can bind to form complexes. We show that this expression and the probability density function $p(r ; R, L)$ which follows from it is also symmetric (invariant) under exchange of $R$ and $L$. Here, we provide explicit expressions for $p(r ; R, L)$ and show that the expression for $r_{e q}$ derived from the reaction rate approach corresponds to $r_{m}$ which maximizes $p(r ; R, L)$, and not to the expectation value $\langle r\rangle$. This indicates that $p(r ; R, L)$ is a skewed distribution, although the skew is not very significant. In Sec. IV, we show that under certain regimes $p(r ; R, L)$, which is a rather complicated expression, can be approximated by a binomial distribution. We then derive the number fluctuations of the bound complex and finally in Sec. V, we connect this problem to a practical situation involving the sensing of biomolecules in the context of the extremely important application of early diagnosis of disease conditions.

\section{THE REACTION RATE APPROACH}

Let us consider a system with $R$ receptors and $L$ ligands. The ligands can bind to the receptors reversibly with binding rate $k_{o n}$ and unbind with a rate $k_{\text {off }}$. The general reaction kinetic equation specifying the rate of formation of complexes $b$ follows from the law of mass action as

$$
\frac{d b}{d t}=k_{o n}(R-b)(L-b)-k_{o f f} b .
$$

Here, the first term in the RHS represents the instantaneous rate of free ligand and free receptor molecules associating to form a complex, while the second term represents the instantaneous rate of dissociation of existing ligand-receptor complexes. At equilibrium, these two opposing reaction rates are equal and the RHS becomes equal to zero from which we see that the number of complexes $b_{e q}$ at equilibrium satisfies the quadratic equation below

$$
k_{\text {on }}\left(R-b_{e q}\right)\left(L-b_{e q}\right)=k_{\text {off }} b_{e q} .
$$


Solving Eq. (2) for $b_{e q}$, we get

$$
b_{e q}=\frac{\left(R+L+K_{D}\right) \pm \sqrt{\left(R+L+K_{D}\right)^{2}-4 R L}}{2}
$$

where $K_{D}=k_{\text {off }} / k_{\text {on }}$.

Equation (3) describes the general solution for the number of bound complexes at steady state indicating two possible values of $b_{e q}$ at equilibrium. However, we know that $b_{e q}$ cannot exceed the maximum of $L$ or $R$. Therefore, the only physically acceptable solution of $b_{e q}$ is

$$
b_{e q}=\frac{\left(R+L+K_{D}\right)-\sqrt{\left(R+L+K_{D}\right)^{2}-4 R L}}{2} .
$$

For special cases, e.g., $R \ll L$ and $R \gg L$, one can modify Eq. (2) by ignoring the depletion in the number of receptor and ligand molecules as shown in Eqs. (5a) and (5b), respectively,

$$
\begin{aligned}
& k_{o n}\left(R-b_{e q}\right) L=k_{o f f} b_{e q}, \\
& k_{o n} R\left(L-b_{e q}\right)=k_{o f f} b_{e q},
\end{aligned}
$$

which gives us the familiar solutions for the reaction kinetic equation known as the Michaelis-Menten equation ${ }^{15}$

$$
\begin{aligned}
& b_{e q(R \ll L)}=\frac{R L}{L+K_{D}}, \\
& b_{e q(R \gg L)}=\frac{R L}{R+K_{D}} .
\end{aligned}
$$

One of the noteworthy differences between Eqs. (4) and (6), besides their different forms, is symmetry with respect to exchange of $R$ and $L$. The form of the solution (Eq. (4)) is symmetric with respect to $R$ and $L$, which is expected given that the receptors and ligands are arbitrary labels. Therefore, interchanging the labels should not affect the final expression. On the other hand, for the special cases (Eq. (6)), the asymmetry comes in because we ignore the depletion of one of the species, thereby distinguishing the labels. A symmetric expression similar to the form of Eq. (6) can be obtained by imposing the condition $4 R L \ll\left(R+L+K_{D}\right)^{2}$ in Eq. (4) as

$$
b_{e q_{(s y m)}}=\frac{R L}{R+L+K_{D}},
$$

which reduces to Eq. (6) for $R \gg L$ or $R \ll L$.

Figure 1 shows the effects of the approximations in Eqs. (6) and (7) in different regimes. To this end, we define relative error $\delta$ as

$$
\delta=\frac{b_{e q}-b_{e q}(\text { approx })}{b_{e q}}
$$

where $b_{e q}$ is the general form of the number of bound receptors described in Eq. (4), and $b_{\text {eq (approx) }}$ represents the three approximated forms for the number of bound receptors, namely, $b_{e q_{(R \ll L)}}, b_{e q(R \gg L)}$, and $b_{e q_{(s y m)}}$. The relative error is plotted with respect to the number of receptor molecules $R$, while the ligand concentration $L$ and the dissociation

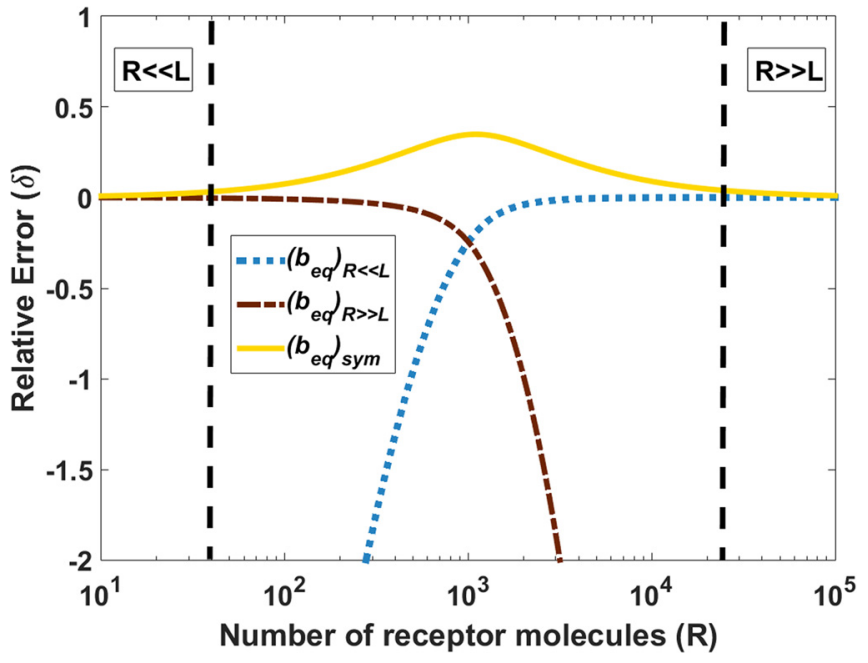

Fig. 1. Effect of approximations: The relative error for the three approximations, namely, $\left(b_{e q}\right)_{R \ll L},\left(b_{e q}\right)_{R \gg L}$, and $\left(b_{e q}\right)_{s y m}$ are plotted with respect to the number of receptors for all the regimes. The symmetric approximation $\left(\left(b_{e q}\right)_{s y m}\right)$ shows the least error across all regimes. The relative error plots of $\left(b_{e q}\right)_{R \ll L}$ and $\left(b_{e q}\right)_{R \gg L}$ do not diverge symmetrically, because the relative error for of $\left(b_{e q}\right)_{R<L}$ becomes independent of $R$ for smaller values (Parameters: $L=1000 ; K_{D}=100$ ).

constant $K_{D}$ are kept constant. Therefore, changing $R$ from $R \ll L$ to $R \gg L$ covers all the possible regimes. As expected, while Eqs. (6a) and (6b) are very good approximations for Eq. (4) for regimes $R \ll L$ and $R \gg L$, respectively, the relative errors for both the approximations are quite significant for other regimes. Interestingly, the symmetric expression $b_{e q_{(s y m)}}$ is the most accurate approximation across all regimes.

\section{PARTITION FUNCTION FOR A SYSTEM CONTAINING $R$ RECEPTORS AND $L$ LIGANDS}

The number of bound complexes at equilibrium can also be calculated from equilibrium statistical mechanics using Gibbs-Boltzmann distribution. As before, we consider a system with $L$ ligands uniformly distributed in the solution and $R$ receptors attached to a surface. We conceptualize the $R$ receptor $L$ ligand problem in a similar manner as the one receptor $L$ ligand problem solved previously. ${ }^{12}$ We discretize the system containing the receptor and ligand molecules in solution as being composed of $\Omega$ boxes or sites as shown in Fig. 2. We imagine that the discretization of the system volume into these boxes is such that any given box or site can be occupied by at most one free ligand or receptor molecule or a single ligand-receptor complex. This means that out of a total of $\Omega$ boxes, $R$ of them would constitute the ones which contain the $R$ receptor molecules present in the system; these are pictorially indicated by the colored boxes in Fig. 2. The ligand molecules, represented by the discs in Fig. 2, can be distributed into any of the total $\Omega$ boxes. Note that a ligand molecule may occupy a colorless (empty) box or colored box. These two options represent the two states that a ligand molecule may be found in, namely, as a free molecule (if occupying a colorless box); and as a ligandreceptor complex (if occupying a colored box). These two states, namely, the free and bound states of the ligand molecule, are associated with energies $\epsilon_{f}$ and $\epsilon_{b}$, respectively, with the sub-scripts $f$ and $b$ denoting the free and bound 


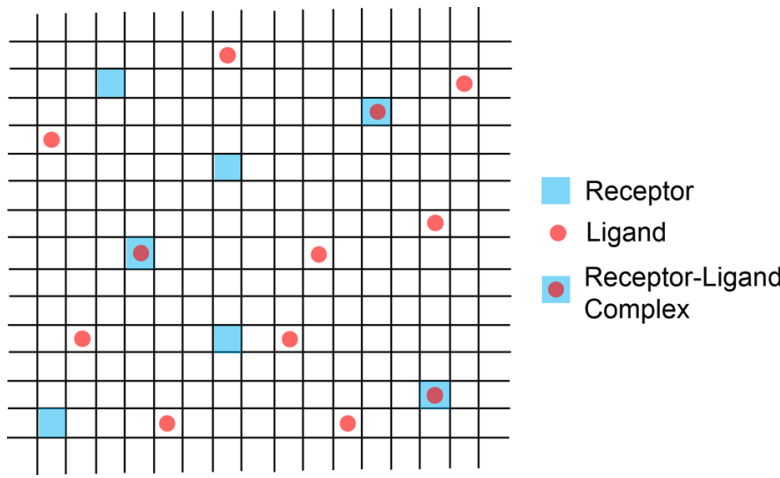

Fig. 2. A schematic representation of the microstates corresponding to a state of the system. The system here is composed of 7 receptors and 12 ligands. A particular configuration corresponding to the state of the system with three ligand-receptor complexes is shown here. Other possible combinations are rearrangements of the objects as described in the main text which are enumerated to obtain the partition function.

states. The system may be found in different configurations each with a different total energy. For example, Fig. 2 shows a specific configuration for a system with $R=7$ and $L=12$ and number of complexes $r=3$. The total energy $E$ of the system with the specific configuration shown in Fig. 2 would be $E=9 \epsilon_{f}+3 \epsilon_{b}$, because out of the 12 ligands, nine are in the free state (occupying colorless boxes) and three are in the bound state (occupying colored boxes, where a receptor is already present). However, as we can clearly see, there can be many more configurations which have the same value of total energy $E$, because we could rearrange the discs into any of the other boxes which are not occupied in Fig. 2. Statistical mechanics provides us with a recipe to calculate the probability of finding the overall state of the system, for instance, with $r=3$ as shown in Fig. 2, from the number of configurations, referred to as microstates, leading to the same overall system state or total energy.

Let us calculate the number of microstates for the state of the system, similar to the one shown in Fig. 2, where the number of receptors, ligands, and complexes are $R, L$, and $r$, respectively. We can do this by calculating the number of ways $L$ indistinguishable ligands can be distributed among the $\Omega-R$ free sites (colorless boxes in Fig. 2) and the $R$ binding sites (colored boxes). The total energy of this state would be $E=(L-r) \epsilon_{f}+r \epsilon_{b}$ as there are $L-r$ ligands which are free and $r$ ligands which are bound, with energy levels $\epsilon_{f}$ and $\epsilon_{b}$, respectively. As already explained in the solution to the one receptor problem, $\Omega \gg R, L$. Therefore, $\Omega-R$ can be well approximated by $\Omega$ itself. Thus, the number of arrangements or configurations of the state of the system with total energy $E=(L-r) \epsilon_{f}+r \epsilon_{b}$ is simply the number of ways to distribute $L-r$ ligands into $\Omega-R \approx \Omega$ free sites and $r$ ligands into the $R$ sites occupied by the receptors, which is simply $\left(\begin{array}{c}\Omega \\ L-r\end{array}\right)\left(\begin{array}{c}R \\ r\end{array}\right)$, where $\left(\begin{array}{l}n \\ k\end{array}\right)$ $=(n ! / \mid k !(n-k) !)$. The partition function $Z$ is then obtained by multiplying this number of microstates with the so-called Boltzmann weight $e^{-\beta E}$, where $\beta=\left(1 / k_{B} T\right)$ with $k_{B}$ being the Boltzmann constant, and summing over all possible values of $r$. The smallest possible value of $r$ is clearly zero (no ligand-receptor complex), and the maximum possible value of $r$ will be the smaller of $R$ and $L$. So the partition function becomes

$$
Z=\sum_{r=0}^{\min (L, R)}\left(\begin{array}{c}
\Omega \\
L-r
\end{array}\right) e^{-(L-r) \beta \epsilon_{f}}\left(\begin{array}{l}
R \\
r
\end{array}\right) e^{-r \beta \epsilon_{b}}
$$

Equation (7) can be further simplified by invoking $\Omega \gg$ $(L-r)$ and therefore

$$
\frac{\Omega !}{(\Omega-(L-r)) !}=\Omega^{L-r} .
$$

Introducing Eq. (9) into Eq. (8), the expression for $Z$ can be simplified as

$$
Z=\left(\Omega e^{-\beta \epsilon_{f}}\right)^{L} \sum_{r=0}^{\min (L, R)} \frac{1}{(L-r) !}\left(\begin{array}{l}
R \\
r
\end{array}\right)\left(\Omega e^{\beta \Delta \epsilon)^{-r}},\right.
$$

where $\Delta \epsilon=\epsilon_{b}-\epsilon_{f}$ is the binding energy of the receptorligand complex.

The probability that there are $r$ bound complexes in the system can be written in terms of the partition function as

$$
p(r ; L, R)=\frac{1}{Z}\left(\frac{\left(\Omega e^{-\beta \epsilon_{f}}\right)^{L}}{(L-r) !}\left(\begin{array}{c}
R \\
r
\end{array}\right)\left(\Omega e^{\beta \Delta \epsilon}\right)^{-r}\right) .
$$

Introducing the expression for $Z$ as derived in Eq. (10) and eliminating the common terms, Eq. (11) gives us

$$
p(r ; L, R)=\frac{\frac{R ! L !}{(R-r) !(L-r) ! r !}\left(\Omega e^{\beta \Delta \epsilon}\right)^{-r}}{\sum_{r^{\prime}=0}^{\min (L, R)} \frac{R ! L !}{\left(R-r^{\prime}\right) !\left(L-r^{\prime}\right) ! r^{\prime} !}\left(\Omega e^{\beta \Delta \epsilon)^{-r^{\prime}}}\right.},
$$

where $r^{\prime}$ is the dummy variable used as the summation index.

Equation (12) describes the general form of $p(r ; L, R)$, which is easily seen to be symmetric with respect to exchange of $L$ and $R$ and hence consistent with the reaction kinetics-based results mentioned in Sec. I. Further, the partition function and the probability distribution shown in Eq. (11) are identical to that obtained from the master equationbased approach as expected. ${ }^{11}$ Obtaining a closed form expression for $p(r ; L, R)$ in Eq. (12) is rather difficult as illustrated in the Appendix. However, we can obtain explicit expressions for $p(r ; L, R)$ for special cases, where $R \gg L$ or $R \ll L$. For instance, take the case where $R \ll L$ as considered commonly in biosensor literature. ${ }^{16-18}$ As $R \ll L$ and maximum of $r$ is the minimum of $L$ and $R$, it follows that $L \gg r$ allowing us to make the approximation:

$$
\frac{L !}{(L-r) !}=L^{r}
$$

Therefore, the partition function for this case $\left(Z_{R \ll L}\right)$ becomes

$$
Z_{R \ll L}=\frac{\left(\Omega e^{-\beta \epsilon_{f}}\right)^{L}\left(1+\alpha_{L}\right)^{R}}{L !},
$$

where $\alpha_{L}=\left(L / \Omega e^{\beta \Delta \epsilon}\right)$.

The probability mass function $p_{R \ll L}(r ; L, R)$ can then be readily obtained as 


$$
p_{R \ll L}(r ; L, R)=\frac{1}{\left(1+\alpha_{L}\right)^{R}}\left[\left(\begin{array}{l}
R \\
r
\end{array}\right) \alpha_{L}^{r}\right] .
$$

Similarly, the probability mass function for $R \gg L$ can be written by interchanging the index $L$ with $R$ in Eq. (12) as

$$
p_{R \gg L}(r ; L, R)=\frac{1}{\left(1+\alpha_{R}\right)^{L}}\left[\left(\begin{array}{l}
L \\
r
\end{array}\right) \alpha_{R}^{r}\right],
$$

where $\alpha_{R}=\left(R / \Omega e^{\beta \Delta \epsilon}\right)$.

Unlike Eq. (12), the expressions in Eqs. (13) and (14) are no longer symmetric with respect to the exchange of $R$ and $L$, because we are ignoring the depletion effect of one of the two species.

By re-arranging the probability mass functions described in Eq. (13) as shown in Eq. (15), we see that $p_{R \ll L}(r ; L, R)$ is a binomial distribution of the form $\left(\begin{array}{l}n \\ k\end{array}\right) p^{k}(1-p)^{n-k}$ with $n=R ; k=r$ and $p=\left(\alpha_{L} / 1+\alpha_{L}\right)$

$$
p_{R \ll L}(r ; L, R)=\left(\begin{array}{l}
R \\
r
\end{array}\right)\left(\frac{\alpha_{L}}{1+\alpha_{L}}\right)^{r}\left(\frac{1}{1+\alpha_{L}}\right)^{R-r} .
$$

The term $(L / \Omega)$ is the concentration of ligand molecules in the solution, and the term $e^{\beta \Delta \epsilon}$ can be mapped to the macroscopic dissociation constant $K_{d}{ }^{12}$ For a system of volume $v$, the dissociation constant $K_{D}$ described in Sec. II can be written in terms of the macroscopic dissociation constant $K_{d}$ as $K_{D}=v K_{d}$. Introducing these relations in Eq. (14), the expression for $p_{R \ll L}(r ; L, R)$ can be obtained as

$$
p_{R \ll L}(r ; L, R)=\left(\begin{array}{l}
R \\
r
\end{array}\right)\left(\frac{L}{L+K_{D}}\right)^{r}\left(\frac{K_{D}}{L+K_{D}}\right)^{R-r} .
$$

The binomial distribution $P(k)=\left(\begin{array}{l}n \\ k\end{array}\right) p^{k}(1-p)^{n-k}$ for $n \rightarrow$ $\infty$ and $p \rightarrow 0$ with the product $n p$ equal to a finite value $\lambda$ can be approximated by a Poisson distribution given by $P(k)=\lambda^{-k} e^{-\lambda} / k !$. Here, the quantity $\lambda$ is the expectation value or the mean of the distribution denoted by $\langle k\rangle$. The number of receptor molecules in many situations is fairly large (of the order of $10^{9}$ or more; see Sec. V, for example) and we may consider $R \rightarrow \infty$. Further if $K_{D} \gg L$, then $p=L / L+K_{D}$ is close to zero. The product of $R$ and $p$ is $\lambda=R L / L+K_{D} \approx R L / K_{D}$. Similarly, for $R \ll L$, one can also show that the above argument is valid if $L \rightarrow \infty$ and $K_{D} \gg R$. Therefore, the Poisson approximation of the binomial distribution will be given by

$$
p_{K_{D} \gg R, L}(r ; L, R)=\frac{\left(\frac{R L}{K_{D}}\right)^{r} e^{-\left(R L / K_{D}\right)}}{r !} .
$$

Equation (17) is commonly used in the case of multistep biological systems such as DNA transcription to simplify the analysis. ${ }^{19}$ The expression is also relevant for describing systems, where the number of bound complexes is extremely low compared to both ligand and receptors, e.g., interaction of non-specific ligand molecules and cell surface receptors. ${ }^{14}$ The steady state distribution described in Eq. (17) can also be derived from the chemical master equation (CME) as described in Ref. 19. To summarize, in this section we showed that under different assumptions, the number of bound complexes at equilibrium, starting from $R$ receptors and $L$ ligands follows different probability distributions. Table I summarizes the analysis of this section.

\section{MOMENTS OF $p(r ; L, R)$}

How are the probability distributions of the number of complexes summarized in Table I related to the number of complexes in Eqs. (3) and (6), derived from the reaction rate approach? To answer this question, we need to calculate the moments of the distributions. Moments are single numbers which characterize an aspect of the distribution, for instance, the first moment (see equation below) of $p(r ; L, R)$ gives us the expectation value or the mean number of bound receptors from the probability mass function. Let us calculate the expectation value for the case $R \ll L$, denoted by the symbol $\langle r\rangle_{R \ll L}$ from the probability mass function provided in Table I. We can write

$$
\begin{aligned}
r_{R \ll L} & =\sum_{r=0}^{R} r p_{R \ll L}(r ; L, R) \\
& =\sum_{r=0}^{R} r\left(\begin{array}{l}
R \\
r
\end{array}\right)\left(\frac{L}{L+K_{D}}\right)^{r}\left(\frac{K_{D}}{L+K_{D}}\right)^{R-r} .
\end{aligned}
$$

This sum can be readily evaluated or one can use the standard result that the mean of the binomial distribution $P(k)$ $=\left(\begin{array}{l}n \\ k\end{array}\right) p^{k}(1-p)^{n-k}$ is $n p$ to yield

$$
\langle r\rangle_{R \ll L}=\frac{R L}{L+K_{D}} .
$$

We see that Eq. (18) is identical to Eq. (6a) obtained from reaction rate approach. Similarly, one can also calculate $\langle r\rangle_{R \gg L}$ by interchanging $L$ and $R$ and see that the expectation value of $r$ from the probability distribution is identical to the number of complexes at equilibrium obtained from the reaction rate.

What about the Poisson approximation mentioned in Eq. (17)? We will only discuss the case $R \ll L$ as the solution to the $R \gg L$ case can be obtained by simply exchanging $R$ and $L$. As discussed in Sec. III, Poisson approximation is valid when $K_{D} \gg L$. This means that the off-rate is significantly high compared to the depletion effect of the ligands, i.e., the ligand-receptor complexes break down into free ligands and free receptors rapidly and therefore, one may assume that $R$ receptors and $L$ ligands are always available for association. The rate of formation of complexes ignoring the depletion of receptors and ligands reads

$$
\frac{d b}{d t}=k_{o n} R L-k_{o f f} b
$$

From which, equating the LHS to zero at equilibrium gives the equilibrium number of complexes as

$$
b_{e q}=\frac{R L}{K_{D}} .
$$

Equation (20) is identical to the mean of the Poisson distribution. Therefore, for all these cases, we see that the mean of the probability distribution of the number of ligand-receptor complexes is identical to the number of ligand-receptor complexes derived from the reaction rate approach. Let us now 
Table I. Landscape of receptor-ligand interactions: We have compiled the results obtained from the binding kinetic equation and equilibrium statistical mechanics for all possible conditions. For the statistical mechanics method, we have the equilibrium probability distribution along with the mean $(r)$ and the mode $\left(r_{m}\right)$ of the distribution which is derived in the article. One interesting point to observe here is that for all the conditions, the expression for the equilibrium solution for the binding kinetic equation $\left(b_{e q}\right)$ is equivalent to the mode $\left(r_{m}\right)$ of the distribution.

\begin{tabular}{|c|c|c|}
\hline Condition & Binding kinetic equation & Equilibrium statistical mechanics \\
\hline$R \ll L$ & $\begin{array}{c}\frac{d b}{d t}=k_{o n}(R-b)(L)-k_{o f f} b \\
b_{e q}=\frac{R L}{L+K_{D}}\end{array}$ & $\begin{array}{c}p_{R \ll L}(r ; L, R)=\left(\begin{array}{c}R \\
r\end{array}\right)\left(\frac{L}{L+K_{D}}\right)^{r}\left(\frac{K_{D}}{L+K_{D}}\right)^{R-r} \\
\langle r\rangle=\frac{R L}{L+K_{D}} \\
r_{m}=\frac{R L}{L+K_{D}} \\
\text { (Binomial distribution) }\end{array}$ \\
\hline$R \gg L$ & $\begin{array}{c}\frac{d b}{d t}=k_{o n}(R)(L-b)-k_{o f f} b \\
b_{e q}=\frac{R L}{R+K_{D}}\end{array}$ & $\begin{array}{c}p_{R \gg L}(r ; L, R)=\left(\begin{array}{c}L \\
r\end{array}\right)\left(\frac{R}{R+K_{D}}\right)^{r}\left(\frac{K_{D}}{R+K_{D}}\right)^{L-r} \\
\langle r\rangle=\frac{R L}{R+K_{D}} \\
r_{m}=\frac{R L}{R+K_{D}} \\
\text { (Binomial distribution) }\end{array}$ \\
\hline$K_{D} \gg R, L$ & $\begin{array}{c}\frac{d b}{d t}=k_{o n} R L-k_{o f f} b \\
b_{e q}=\frac{R L}{K_{D}}\end{array}$ & $\begin{aligned} & p_{K_{D} \gg R, L}(r ; L, R)=\frac{\left(\frac{L R}{K_{D}}\right)^{r} e^{-\left(L R / K_{D}\right)}}{r !} \\
&\langle r\rangle= \frac{R L}{K_{D}} \\
& r_{m}=\frac{R L}{K_{D}} \\
& \text { (Poisson distribution) }\end{aligned}$ \\
\hline \multirow[t]{3}{*}{$R \approx L$} & $\frac{d b}{d t}=k_{\text {on }}(R-b)(L-b)-k_{\text {off }} b$ & $\frac{R ! L !}{(R-r) !(L-r) ! r !}\left(\Omega e^{\beta \Delta \epsilon}\right)^{-r}$ \\
\hline & $b_{e q}=\frac{\left(R+L+K_{D}\right)-\sqrt{\left(R+L+K_{D}\right)^{2}-4 R L}}{2}$ & $\begin{array}{c}p(r ; L, R)=\frac{\sum_{r^{\prime}=0}^{\min (L, R)} \frac{R ! L !}{\left(R-r^{\prime}\right) !\left(L-r^{\prime}\right) ! r^{\prime} !}\left(\Omega e^{\beta \Delta \epsilon}\right)^{-r^{\prime}}}{U\left(-L,-L+R+1,-K_{D}\right)} \\
\langle r\rangle=L+\frac{K_{D} L U\left(1-L,-L+R+2,-K_{D}\right)}{U(-L)}\end{array}$ \\
\hline & & $r_{m}=\frac{\left(R+L+K_{D}\right)-\sqrt{\left(R+L+K_{D}\right)^{2}-4 R L}}{2}$ \\
\hline
\end{tabular}
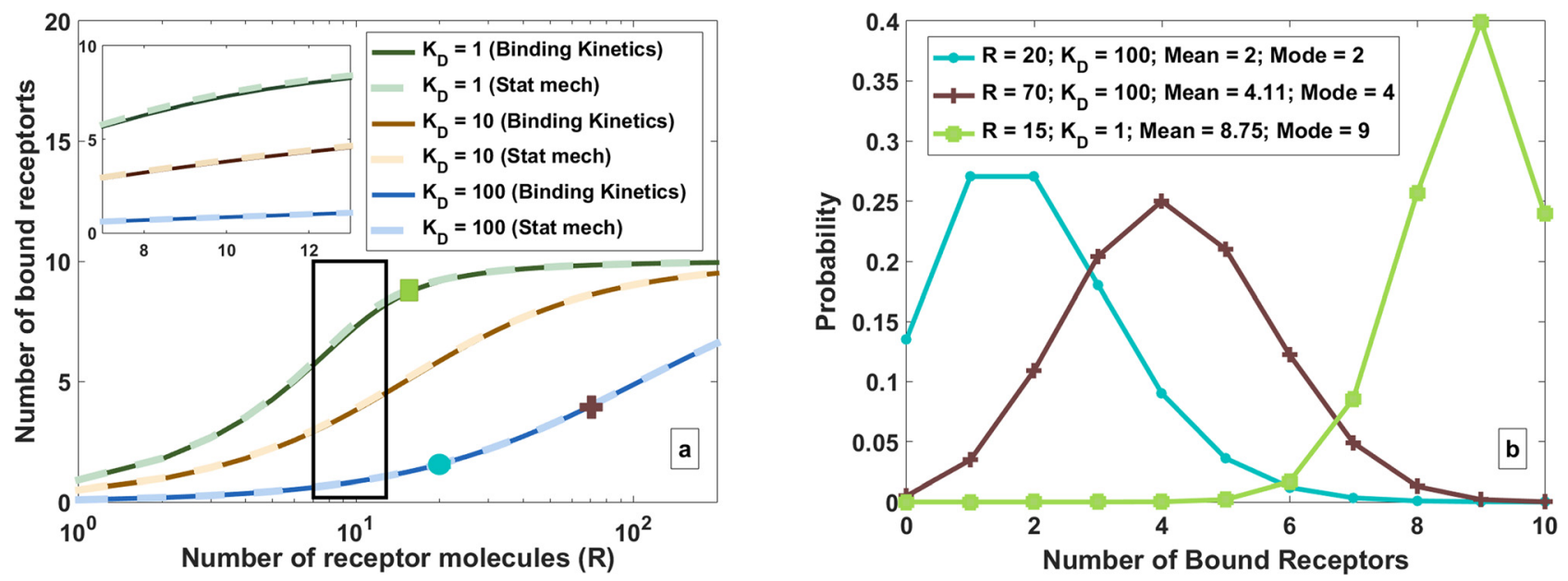

Fig. 3. (a) Comparison of the general forms of the solution obtained from the binding kinetic equation and equilibrium statistical mechanics. For special cases, i.e., $|L-R| \gg 0$, both the solutions match perfectly. However, for $L \approx R$, there is some difference between them as shown in the subfigure, particularly for low values of $K_{D}$. The difference reduces with an increase in $K_{D}$. (b) Probability distribution for different conditions, which are shown by coloured circles in Fig. 3(a). Irrespective of the shape of the distribution, the mean and mode are very close to each other (Parameters: $L=10)$. 
calculate the mean of the distribution for the general case described in Eq. (12).

For the general case, the expression for the mean number of bound complexes can be written as

$$
\langle r\rangle=\sum_{r=0}^{\min (L, R)} r p(r ; L, R)=\frac{\sum_{r=0}^{\min (L, R)} \frac{r R ! L !}{(R-r) !(L-r) ! r !}\left(\Omega e^{\beta \Delta \epsilon}\right)^{-r}}{\sum_{r=0}^{\min (L, R)} \frac{R ! L !}{(R-r) !(L-r) ! r !}\left(\Omega e^{\beta \Delta \epsilon}\right)^{-r}} .
$$

From the calculations described in the Appendix, $\langle r\rangle$ can be written as

$$
\langle r\rangle=L+\frac{K_{D} L U\left(1-L,-L+R+2,-K_{D}\right)}{U\left(-L,-L+R+1,-K_{D}\right)},
$$

where $U(a, b, z)$ is the confluent hypergeometric function of the second kind. Interestingly, the form of Eq. (22) is quite different from the result obtained using the rate equation in Eq. (3). In Fig. 3(a), we have plotted the expression for $\langle r\rangle$ from Eq. (22) and the number of equilibrium ligand-receptor complexes from the reaction rate approach from Eq. (3) to numerically compare them. It is clear that while these expressions are very different algebraically, they match each other quite well numerically. The two expressions diverge marginally for $L \approx R$.

While the number of ligand-receptor complexes calculated using reaction kinetics approach numerically matches the expectation value obtained from the probability mass function of the number of complexes, the algebraic expressions are very different. Is there any other quantity derivable from the probability mass function of the ligand-receptor complexes which exactly matches the solution form the reaction rate approach? Indeed, we see that the mode of $p(r ; L, R)$, denoted by $r_{m}$, exactly matches Eq. (3) derived from the reaction rate approach. To calculate the mode of a distribution, we need to maximize the distribution with respect to $r$. We maximize $\log (p(r ; L, R))$ taking advantage of the monotonicity of the $\log$ function.

Introducing log in Eq. (11), we get

$$
\begin{aligned}
\log p(r ; L, R)= & \log \left(\frac{R ! L !}{Z}\right)-\log ((R-r) !) \\
& -\log ((L-r) !)-\log (r !) \\
& -r \log \left(\Omega e^{\beta \Delta \epsilon}\right) .
\end{aligned}
$$

Using Stirling's approximation

$$
\log x !=x \log x-x
$$

in Eq. (23) and maximizing with respect to $r$ gives us

$$
\log \left(\frac{\left(L-r_{m}\right)\left(R-r_{m}\right)}{r_{m} \Omega e^{\beta \Delta \epsilon}}\right)=0,
$$

where $r_{m}$ is the mode of the probability distribution. As explained before, $\Omega e^{\beta \Delta \epsilon}$ can be mapped to the dissociation constant $K_{D}$ described in Sec. II. Therefore, Eq. (24) becomes

$$
\left(L-r_{m}\right)\left(R-r_{m}\right)=r_{m} K_{D},
$$

which can be solved for $r_{m}$ to obtain

$$
r_{m}=\frac{\left(R+L+K_{D}\right) \pm \sqrt{\left(R+L+K_{D}\right)^{2}-4 R L}}{2} .
$$

Similar to $r_{e q}$, the upper bound of $r_{m}$ is $\max (L, R)$. Therefore, we only consider the negative quadratic to be the physically acceptable solution. Therefore, $r_{m}$ becomes

$$
r_{m}=\frac{\left(R+L+K_{D}\right)-\sqrt{\left(R+L+K_{D}\right)^{2}-4 R L}}{2} .
$$

Therefore, the number of bound receptors at steady state $b_{e q}$ derived in Sec. I is the number of bound receptors one is expected to obtain experimentally with maximum probability and not the average number of bound receptors. In other words, the maximum probable state with $r_{m}$ number of complexes is precisely the equilibrium state of this system. Why does the number of bound complexes obtained from reaction rate equations match the expected value $\langle r\rangle$ obtained from the probability distribution function $p(r ; L, R)$ numerically even though their analytical expressions are quite different? To answer this, we note that all the distributions in Table I, including special cases such as $R \ll L$, have their mode close to the mean irrespective of asymmetries in the distributions. Figure 3(b) illustrates the point mentioned above.

\section{HIGHER MOMENTS OF $p(r ; L, R)$}

One advantage of the equilibrium statistical mechanics method over the reaction rate approach is that it allows us to calculate all the moments of the probability distribution function at equilibrium. Having established that $p_{R \ll L}(r ; L, R)$ is the binomial distribution for the special case of $R \ll L$ considered here, we can readily find the variance in the number of bound complexes, $\sigma_{R \ll L}^{2}$, as

$$
\sigma_{R \ll L}^{2}=\frac{R L K_{D}}{\left(L+K_{D}\right)^{2}} .
$$

Similarly, for $R \gg L$, the variance can be obtained by interchanging $L$ and $R$ in Eq. (28).

Although obtaining closed form expressions for the higher moments for the general form of $p(r ; L, R)$ is not possible, we can obtain a general expression for the higher moments.

To estimate the higher moments, we define a moment generating function

$$
G(z)=\sum_{r=0}^{\min (L, R)} z^{r} p(r ; L, R) .
$$

The $n^{\text {th }}$ moment $M(n)$ of $p(r ; L, R)$ can be obtained by calculating the $n^{\text {th }}$ partial differential of $G(z)$ at $z=1$. The $n^{\text {th }}$ partial differential of $G(z)$ at $z=1$ gives us

$$
\left.\partial_{z}^{n} G(z)\right|_{z=1}=\sum_{r=0}^{\min (L, R)}\left(\prod_{i=0}^{n}(r-i) p(r ; L, R)\right) .
$$

Expanding Eq. (30), we get 


$$
\begin{aligned}
\left.\partial_{z}^{n} G(z)\right|_{z=1}= & \sum_{r=0}^{\min (L, R)} p(r ; L, R)\left(r^{n}-\sum_{a=1}^{r-1} a r^{n-1}\right. \\
& \left.+\sum_{b=1}^{r-1} \sum_{c>b}^{r-1} b c r^{n-2} \ldots \ldots\right) .
\end{aligned}
$$

In the literature, the coefficient of $r^{k}$ in Eq. (31) is called Stirling's number of the first kind and is denoted as $s(n, k){ }^{20}$ Therefore, Eq. (31) can be rewritten in terms of Stirling's numbers as

$$
\left.\partial_{z}^{n} G(z)\right|_{z=1}=\sum_{r=0}^{\min (L, R)} p(r ; L, R) \sum_{k=0}^{n} s(n, k) r^{k} .
$$

The $n^{\text {th }}$ moment $M(n)$ now can be calculated as

$$
M(n)=\left.\partial_{z}^{n} G(z)\right|_{z=1}-\left.\sum_{i=1}^{n-1} \rho(n-i) \partial_{z}^{i} G(z)\right|_{z=1},
$$

where $\rho(k)=s(n, k)-\sum_{i=1}^{k-1} \rho(i) s(n-i, k-i)$,with $\rho(0)=1$, and $\rho(1)=s(n, 1)$.

\section{A NUMERICAL EXAMPLE: MICRO/NANO PARTICLE-BASED MOLECULAR SENSORS}

Early, pre-symptomatic, detection of cancer is an unmet demand which can potentially save millions of lives annually. ${ }^{21}$ Detection of a class of molecules called cytokines has been pursued as a means to achieve early detection of cancer. ${ }^{22}$ In such sensors, a sample volume $V_{s}$ containing the target ligands interacts with a system containing receptors, and the number of bound complexes translates into a physically measurable quantity such as a change in electrical conductance or optical absorbance. ${ }^{23}$ For a designer of such a molecular sensing system, it is important to understand the effect of design choices such as the area of the sensing region used to capture target ligand molecules from the sample, the volume of the sample to be used, etc., on the noise due to the stochastic nature of molecular binding. Using what we learned about the variance of molecular binding in Sec. V, we can begin to answer such questions, an example of which is provided below.

Let us consider a sensor system, shown schematically in Fig. 4, consisting of a sensor surface of radius $a$, where receptor molecules are attached. Typically, the surface of the particle is saturated with a monolayer of receptor molecules. Therefore, the number of receptor molecules on each bead can be estimated to be $R=\pi a^{2} / d^{2}$, where $d$ is the size of a

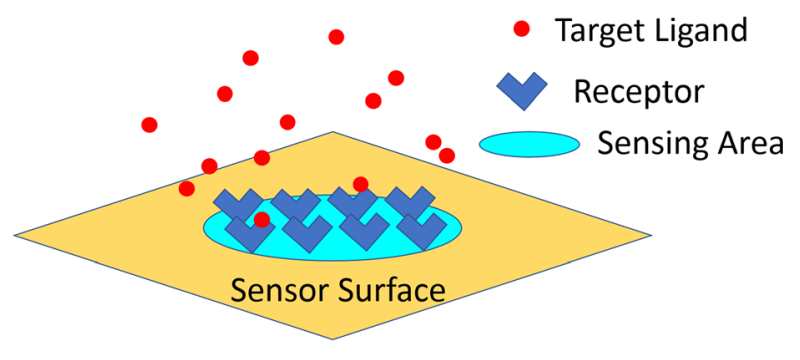

Fig. 4. Schematic representation of a molecular sensing device. Receptors immobilized in a sensing area bind target ligands in a sample. single receptor molecule which we can take to be of the order of $10 \mathrm{~nm}^{24}$ The number of ligand molecules is $L=c V_{s}$, where $c$ is the concentration of ligand molecules in the sample. Given these conditions, how should one choose the area of the sensing region (size $a$ in Fig. 4), so that the fluctuation in the number of the bound receptors is less than $1 \%$ of the average number of the bound receptors?

For a real system, $V_{s}$ is typically of the order of $100 \mu$ l. Taking $c$ to be 10 pico-molar (pM), the number of ligand molecules in the system becomes $6 \times 10^{8}$. The mean number of bound receptors, $\mu$, and the variance in the number of bound receptors, $\sigma^{2}$, on the sensing region can be obtained as

$$
\begin{aligned}
& \mu=\frac{R L}{L+K_{D}}, \\
& \sigma^{2}=\frac{R L K_{D}}{\left(L+K_{D}\right)^{2}} .
\end{aligned}
$$

We define the relative fluctuation per particle as $\sigma_{\mathrm{REL}}=\sigma / \mu$ as

$$
\sigma_{\mathrm{REL}}=\sqrt{\frac{K_{D}}{R L}}
$$

Substituting the expression for $R$ and $L$ from above, we get

$$
\sigma_{\mathrm{REL}}=\sqrt{\frac{K_{D} d^{2}}{\pi a^{2} c V_{s}}} .
$$

We take the value of $K_{D}$ to be around $6 \times 10^{9}$, corresponding to a dissociation constant of $100 \mathrm{pM}$. If the desired fluctuation of the bound number of receptors (which will generate the sensor signal) is to be less than $1 \%$, from Eq. (36), we see that

$$
a>\sqrt{\frac{K_{D} d^{2}}{\pi \sigma_{R E L} c V_{s}}} .
$$

Putting the numerical values we see that the size of the sensing region must be greater than about $200 \mathrm{~nm}$ to achieve a relative fluctuation of less than $1 \%$ in the number of bound receptors. Why does this happen? This is because reducing the size of the sensing region decreases the number of receptors available in the sensing region and increases the relative fluctuations as seen from Eq. (36). This exercise immediately tells us that extreme miniaturization as seen in single-molecule-level sensors such as those based on nanopores or nano field-effect transistors ${ }^{25,26}$ are likely to suffer from large relative fluctuations due to the small number of receptors available.

\section{CONCLUSIONS}

In this article, we derived the equilibrium probability distribution of the number of ligand-receptor complexes in a system consisting of $R$ receptors and $L$ ligands. This work is an extension of a similar problem with one receptor and $L$ ligands, which is now widely available in the references provided in this article. However, a treatment of the general $R$ receptor case and the thorough description of the connections between equilibrium probability distributions and their 
connections with the number of complexes obtained using the reaction rate approach under various special cases is lacking in the current literature. We hope that this article has addressed this gap to some extent. For instance, in Table I, we summarized different situations relevant to molecular sensing and compared the equilibrium statistical mechanics and the reaction rate approaches and showed how these relate to one another, thus allowing the student to appreciate the equivalence of these methods. Further, this article helped us to illustrate two aspects of this problem which are not directly apparent in the solution to the single receptor problem, namely, (a) a symmetry of the equilibrium probability distribution of the number of bound complexes under exchange of $R$ and $L$ and (b) the number of bound complexes obtained from chemical kinetic equations has an exact correspondence to the maximum probable value of $r$. Overall, we hope that this article introduces the application of statistical mechanics to molecular binding problems in a broader setting than previous work.

\section{APPENDIX: EXPRESSION FOR $p(r ; L, R)$ IN THE GENERAL CASE}

From Abramowitz and Stegun ${ }^{20}$ Eq. (13.5.2), we obtain

$$
U(a, b, z)=z^{-a}\left\{\sum_{r=0}^{T-1} \frac{(a)_{r}(1+a-b)_{r}}{r !}(-z)^{-r}+O\left(|z|^{-T}\right)\right\} .
$$

Here, $U(a, b, z)$ is the confluent hypergeometric function of the second kind, and $(x)_{n}$ is the Pochhammer symbol defined as

$$
(x)_{n}=\frac{\Gamma(x+n)}{\Gamma(x)},
$$

where $\Gamma(x)$ is the Gamma function.

The summation in Eq. (A1) is equivalent to the partition function $Z$ defined in Eq. (9) for $T=\min (L, R)+1, a=-L$, $b=1+R-L$, and $z=-K_{D}$. The error term can be neglected for $K_{D} \gg 0$ or $\min (L, R) \gg 0$. Introducing the parameters in Eq. (A1) and using the property

$$
(-x)_{n}=(-1)^{n}(x-n+1)_{n},
$$

we obtain

$$
\begin{aligned}
Z & =\sum_{r=0}^{\min (L, R)}\left(\frac{L !}{(L-r) ! r !}\right)\left(\frac{R !}{(R-r) !}\right)\left(K_{D}\right)^{-r} \\
& =\left(-K_{D}\right)^{-L} U\left(-L, 1+R-L,-K_{D}\right) .
\end{aligned}
$$

To derive the expression for the mean of the distribution $(\mu)$, we use the moment generating function $G(\eta)$ defined in Eq. (28) in the main text, which becomes

$$
G(\eta)=\eta^{L} \frac{U\left(-L, 1+R-L,-\frac{K_{D}}{\eta}\right)}{U\left(-L, 1+R-L,-K_{D}\right)} .
$$

$\mu$ can now be obtained from Eq. (A3) as

$$
\mu=\left.\frac{\partial G(\eta)}{\partial \eta}\right|_{\eta=1}
$$

Using the relation ${ }^{20}$

$$
\frac{d}{d z} U(a, b, z)=-a U(a+1, b+1, z)
$$

we obtain $\mu$ as

$$
\mu=L+\frac{K_{D} L U\left(1-L,-L+R+2,-K_{D}\right)}{U\left(-L,-L+R+1,-K_{D}\right)} .
$$

${ }^{1}$ J. T. Margaret, B. K. Chu, M. Roy, and E. L. Read, "Dna-binding kinetics determines the mechanism of noise-induced switching in gene networks," Biophys. J. 109(8), 1746-1757 (2015).

${ }^{2}$ P. A. González, L. J. Carreño, D. Coombs, J. E. Mora, E. Palmieri, B. Goldstein, S. G. Nathenson, and A. M. Kalergis, "T cell receptor binding kinetics required for $t$ cell activation depend on the density of cognate ligand on the antigen-presenting cell," Proc. Natl. Acad. Sci. U. S. A. 102(13), 4824-4829 (2005).

${ }^{3}$ R. Phillips, "Napoleon is in equilibrium," Annu. Rev. Condens. Matter Phys. 6(1), 85-111 (2015).

${ }^{4}$ A. B. Koudriavtsev, R. F. Jameson, and W. Linert, The Law of Mass Action (Springer Science \& Business Media, Berlin, 2011).

${ }^{5}$ K. Ghosh, K. A. Dill, M. M. Inamdar, E. Seitaridou, and R. Phillips, "Teaching the principles of statistical dynamics," Am. J. Phys. 74(2), 123-133 (2006).

${ }^{6}$ R. G. Endres, Physical Principles in Sensing and Signaling: With an Introduction to Modeling in Biology (Oxford U. P., Oxford, 2013).

${ }^{7}$ T. Mora, "Physical limit to concentration sensing amid spurious ligands," Phys. Rev. Lett. 115(3), 038102 (2015).

${ }^{8}$ P. R. Ten Wolde, N. B. Becker, T. E. Ouldridge, and A. Mugler, "Fundamental limits to cellular sensing," J. Stat. Phys. 162(5), 1395-1424 (2016).

${ }^{9}$ J. V. Bonventre and V. S. Vaidya, Biomarkers: In Medicine, Drug Discovery, and Environmental Health (Wiley, New Jersey, 2010).

${ }^{10}$ N. G. Van Kampen, Stochastic Processes in Physics and Chemistry (Science Direct, Singapore, 2007).

${ }^{11} \mathrm{~K}$. Ghosh, "Stochastic dynamics of complexation reaction in the limit of small numbers," J. Chem. Phys. 134(19), 05 B606 (2011).

${ }^{12}$ H. G. Garcia, J. Kondev, N. Orme, J. A. Theriot, and R. Phillips, “A first exposure to statistical mechanics for life scientists," preprint arXiv:0708.1899.

${ }^{13}$ F. Bai, X. Pi, P. Li, P. Zhou, H. Yang, X. Wang, M. Li, Z. Gao, and H. Jiang, "A statistical thermodynamic model for ligands interacting with ion channels: Theoretical model and experimental validation of the kcnq2 channel," Front. Pharmacol. 9, 150 (2018).

${ }^{14}$ D. A. Lauffenburger and J. J. Linderman, Receptors: Models for Binding, Trafficking, and Signaling (Oxford U. P., Oxford, 1996).

${ }^{15}$ A. Cornish-Bowden, Fundamentals of Enzyme Kinetics (John Wiley \& Sons, New Jersey, 2013).

${ }^{16} \mathrm{~S}$. De Picciotto, B. Imperiali, L. G. Griffith, and K. D. Wittrup, "Equilibrium and dynamic design principles for binding molecules engineered for reagentless biosensors," Anal. Biochem. 460, 9-15 (2014).

${ }^{17}$ T. M. Squires, R. J. Messinger, and S. R. Manalis, "Making it stick: Convection, reaction and diffusion in surface-based biosensors," Nat. Biotechnol. 26(4), 417-426 (2008).

${ }^{18}$ B. Saha, T. H. Evers, and M. W. Prins, "How antibody surface coverage on nanoparticles determines the activity and kinetics of antigen capturing for biosensing," Anal. Chem. 86(16), 8158-8166 (2014).

${ }^{19}$ S. Iyer Biswas, "Applications of methods of non-equilibrium statistical physics to models of stochastic gene expression," Ph.D. dissertation, The Ohio State University, 2009.

${ }^{20}$ M. Abramowitz and I. Stegun, Handbook of Mathematical Functions with Formulas, Graphs, and Mathematical Tables, June 1964, National Bureau of Standards Applied Mathematics Series Vol. 55 (National Bureau of Standards, Maryland, 1964), pp. 446-449. 
${ }^{21}$ See $<$ https://www.cancer.gov/about-cancer/understanding/statistics $>$ for statistics of different types of cancers and their survival rates in the United States and around the world.

${ }^{22}$ Y. Cai, J. Zhu, J. He, W. Yang, C. Ma, F. Xiong, F. Li, W. Chen, and P. Chen, "Magnet patterned superparamagnetic fe3o4/au core-shell nanoplasmonic sensing array for label-free high throughput cytokine immunoassay," Adv. Healthcare Mater. 8(4), 1801478 (2019).

${ }^{23}$ L. Cohen and D. R. Walt, "Highly sensitive and multiplexed protein measurements,” Chem. Rev. 119(1), 293-321 (2018).
${ }^{24}$ M. A. G. de Castro, C. Höbartner, and F. Opazo, “Aptamers provide superior stainings of cellular receptors studied under super-resolution microscopy," PLoS One 12(2), e0173050 (2017).

${ }^{25}$ P. Waduge, R. Hu, P. Bandarkar, H. Yamazaki, B. Cressiot, Q. Zhao, P. C. Whitford, and M. Wanunu, "Nanopore-based measurements of protein size, fluctuations, and conformational changes," ACS Nano 11(6), 5706-5716 (2017).

${ }^{26}$ M. Kaisti, "Detection principles of biological and chemical fet sensors," Biosens. Bioelectron. 98, 437-448 (2017).

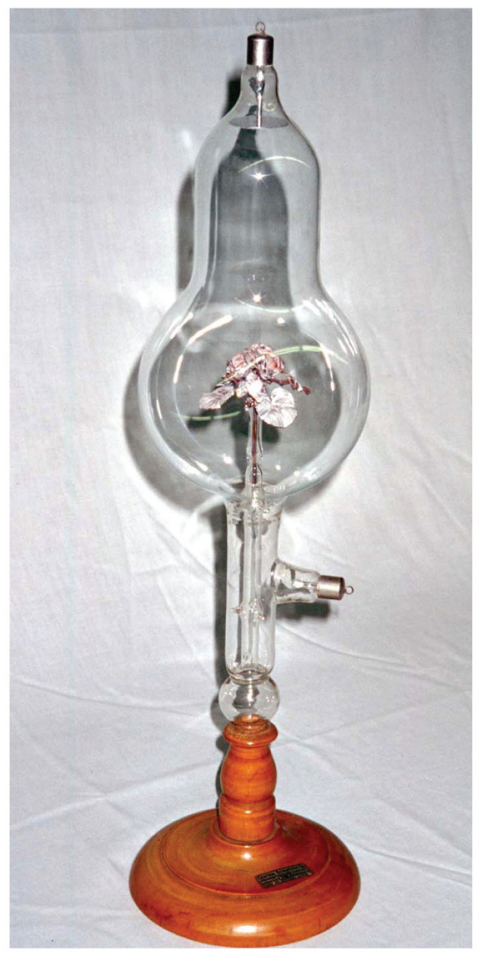

\section{Crooke's Tube with Bouquet}

Most Crooke's tubes were used to demonstrate the properties of cathode rays. Others were made for the visual enjoyment of natural phenomena. This tube, in the collection of Hobart and William Smith Colleges, is one of the latter. This tube has a spray of flowers in the middle, made of mica and covered with fluorescent paint. The electron stream striking the leaves made the flower fluoresce. This example was made by Max Kohl of Chemnitz, Germany. In the 1900 Kohl catalogue there is a similar tube, but with a radiometer, spun by the beam, mounted above the flowers. This apparatus was photographed at Hobart and William Smith Colleges. (Picture and text by Thomas B. Greenslade, Jr., Kenyon College) 